\title{
A frameshift mutation in the TRPS1 gene showing a mild phenotype of trichorhinophalangeal syndrome type 1
}

\author{
Jin-Mo Park', Yun Jeong Lee', and Jin-Sung Park ${ }^{3, *}$ \\ ${ }^{1}$ Department of Neurology, Dongguk University College of Medicine, Gyeongju, Korea \\ ${ }^{2}$ Department of Pediatric Neurology, Kyungpook National University Hospital, Daegu, Korea \\ ${ }^{3}$ Department of Neurology, School of Medicine, Kyungpook National University Chilgok Hospital, Daegu, Korea
}

Tricho-rhino-phalangeal syndrome (TRPS) is a hereditary disorder characterized by craniofacial and skeletal abnormalities. A mutation of the TRPS1 gene leads to TRPS type I or type III. A 20-year-old male patient visited our neurologic department with chronic fatigue. He presented with short stature, sparse hair, pear-shaped nose, and brachydactyly. Radiologic study showed short metacarpals, metatarsals with cone-shaped epiphyses, hypoplastic femur and hip joint. Panel sequencing for OMIM (Online Mendelian Inheritance in Man) listed genes revealed a de novo heterozygous frameshift mutation of c.1801_1802delGA (p.Arg601Lysfs*3) of exon 4 of the TRPS1 gene. The diagnosis of TRPS can be challenging due to the rarity and variable phenotype of the disease, clinicians should be aware of its characteristic clinical features that will lead a higher rate of diagnosis.

Key words: Trichorhinophalangeal syndrome, Genes, Phenotype.

\section{Introduction}

Tricho-rhino-phalangeal syndrome (TRPS) is an autosomal dominant genetic disorder characterized by craniofacial and skeletal abnormalities [1]. To date, three subtypes of TRPS have been reported: TRPS type I (TRPS1; MIM 190350), TRPS type II (TRPS2; MIM 150230), and TRPS type III (TRPS3; MIM 190351). Prevalence of TRPS is estimated as 0.2 to 1 per 100,000, while unbiased population-based estimates of the prevalence of TRPS are not available [2]. Patients with TRPS1 clinically show sparse scalp hair, bulbous tip nose, thin upper lip, brachydactyly, coneshaped epiphysis, and protruding ears. Patients with TRPS3 present with similar clinical features that are clinically similar to TRPS1, but usually show relatively severe skeletal abnormalities [1]. The TRPS 1 gene is mutated in both TRPS1 and TRPS3 subtypes, and the TRPS1 gene is known to encode zinc finger transcriptional repressor which regulates the development of chondrocytes and perichondrium [3]. Patients with TRPS2 caused by contiguous microdeletions involving both TRPS1 and EXT1 genes show multiple exostoses and variable degrees of mental retardation, that differentiates from TRPS1 and 3 [4]. We describe herein a Korean adult case of TRPS1 in which we have identified a novel pathogenic mutation in the TRPS1 gene by panel sequencing using next generation sequencing technology.

Received: 18 May 2018, Revised: 25 June 2018, Accepted: 26 June 2018, Published: 31 December 2018

${ }^{*}$ Corresponding author: Jin-Sung Park, M.D. (D) http://orcid.org/0000-0001-5506-9206

Department of Neurology, School of Medicine, Kyungpook National University Chilgok Hospital, 807 Hoguk-ro, Buk-gu, Daegu 41404, Korea.

Tel: +82-53-200-2753, Fax: +82-53-200-2029, E-mail: neurojspark@gmail.com

Conflict of interest: The authors declare that they do not have any conflicts of interest.

(c) This is an open-access article distributed under the terms of the Creative Commons Attribution Non-Commercial License (http://creativecommons.org/licenses/by-nc/4.0/) which permits unrestricted non-commercial use, distribution, and reproduction in any medium, provided the original work is properly cited.

(c) Copyright 2018 by the Korean Society of Medical Genetics and Genomics 


\section{Case}

A 20-year-old male patient visited our clinic complaining of malaise and fatigue. He had no past history of delay in the milestones with a height of $163 \mathrm{~cm}$ (-1.9 standard deviation). The patient showed typical appearance of a receding frontal hairline, sparse hair on vertex area, sparse lateral eyebrows and pear-shaped nose. Physical examination revealed shortness of both thumbs and great toes that were consistent with brachydactyly (Fig. 1). When he was young, the patient had thought he had thin hair, but at the age of 18 he developed sparse hair. A local dermatologist diagnosed him as androgenetic alopecia at the age of 19. He was on finasteride to treat alopecia for more than a year that turned out to be ineffective. Considering his educational background, his intelligence seemed to be normal. One year ago, he underwent reconstructive surgery caused by temporomandibular joint derangement. Neurologic examination, electrophysiologic and laboratory studies were performed to reveal the cause of malaise and fatigue. Despite of subjective weakness and fatigue, his motor power was full with normoactive deep tendon reflexes. Nerve conduction study and electromyography did not reveal any abnormalities. Laboratory tests including thyroid function, growth hormone and insulin growth factor $(289 \mathrm{ng} / \mathrm{mL}$ ) were within normal limits. The X-rays showed short metacarpals, metatarsals with cone-shaped epiphysis; hypoplastic femur and hip joint (Fig. 2). The dual-energy X-ray absorptiometry of femur and hip bones showed a T score of -1.4 reflecting osteopenia. Informed consent was obtained and a blood sample was taken from the patient in order to analyze the DNA through the Illumina NextSeq 500 platform (Illumina, Inc., San Diego, CA, USA) and an enrichment protocol, Trusight One Sequencing Panel (Illumina, Inc.) for the simultaneous sequencing of the exon regions of 4,813 clinically relevant genes (GC genome, Yongin, Korea). This led to the identification of novel heterozygous frameshift mutation of c.1801_1802delGA (p.Arg601 Lysfs*3) of exon 4 of the TRPS 1 gene, which was confirmed by Sanger sequencing (Fig. 3). Neither parents nor younger brother had this mutation, reflecting the de novo mutation in the TRPS1 gene of the patient. Combination drug of calcium and vitamin D were prescribed to the patient, and he is on regular follow up.

\section{Discussion}

Typical clinical features of TRPS 1 include growth impairment, sparse hair, long philtrum, broad nasal ridge, short metacarpals and brachydactyly. According to a recent cohort study the morbidity of TRPS was closely related to decreased mobility, joint
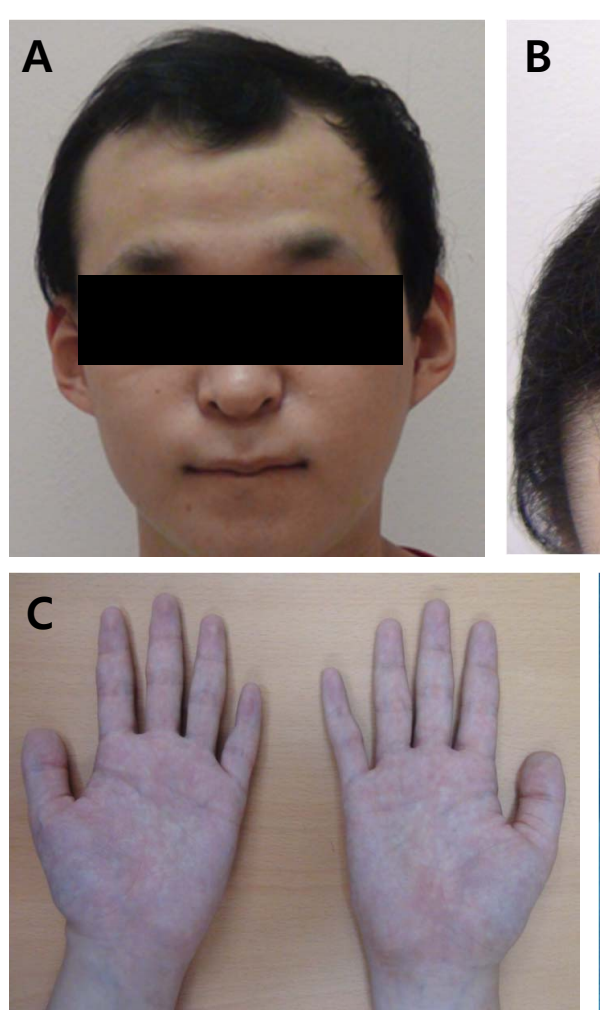

Fig. 1. The clinical features of trichorhino-phalangeal syndrome include sparse eyebrow, broad nasal tip, and broad nasal ridge $(A)$, receding frontal hairline and sparse hair on vertex area (B), shortness of both thumbs $(C)$, and great toes $(D)$. 

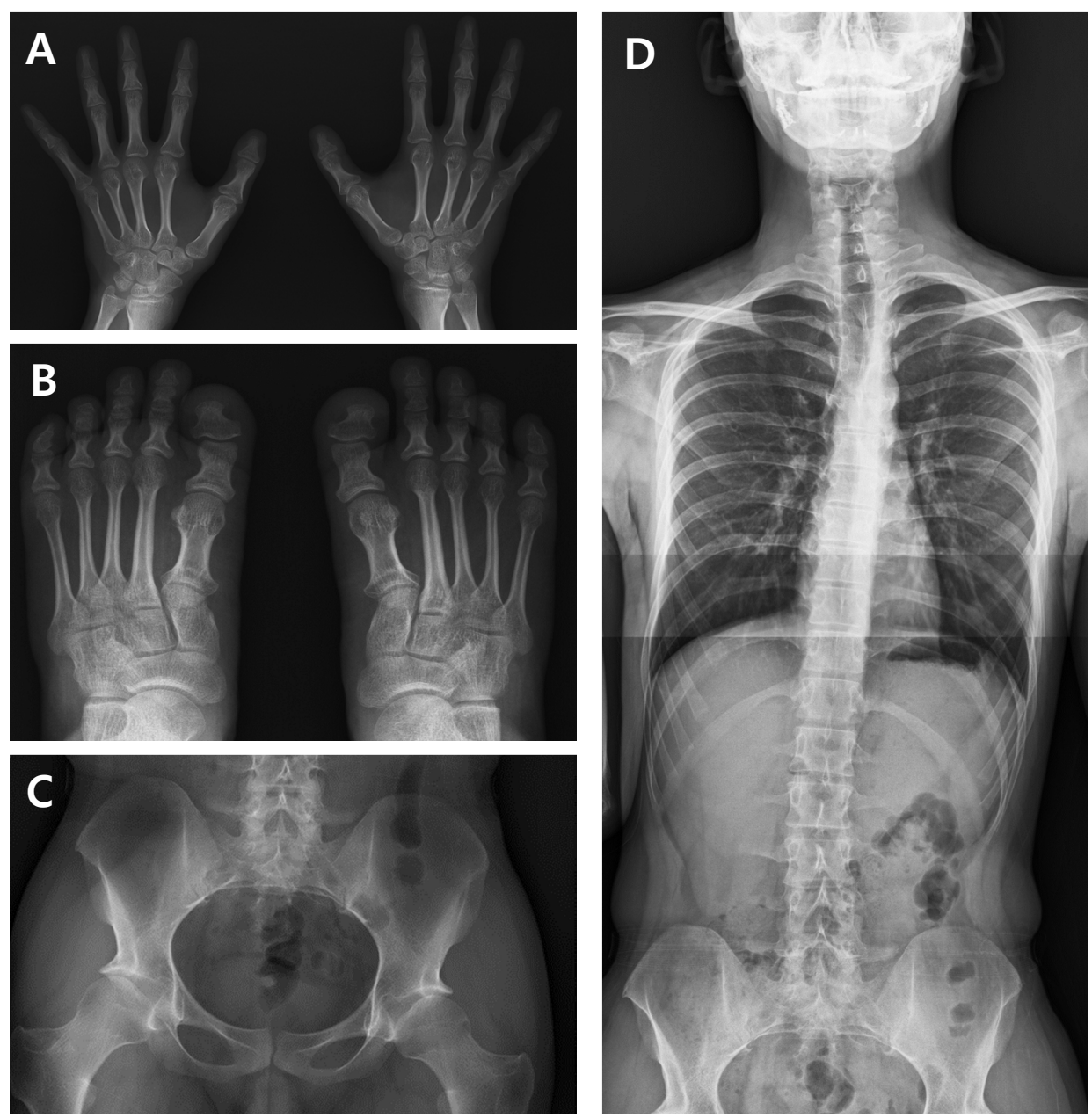

Fig. 2. Radiologic findings of skeletal abnormalities of the TRPS1 patient that show cone-shaped epiphyses in the distal limbs $(A, B)$, hypoplastic femur and hip joint $(C)$ and scoliosis (D).
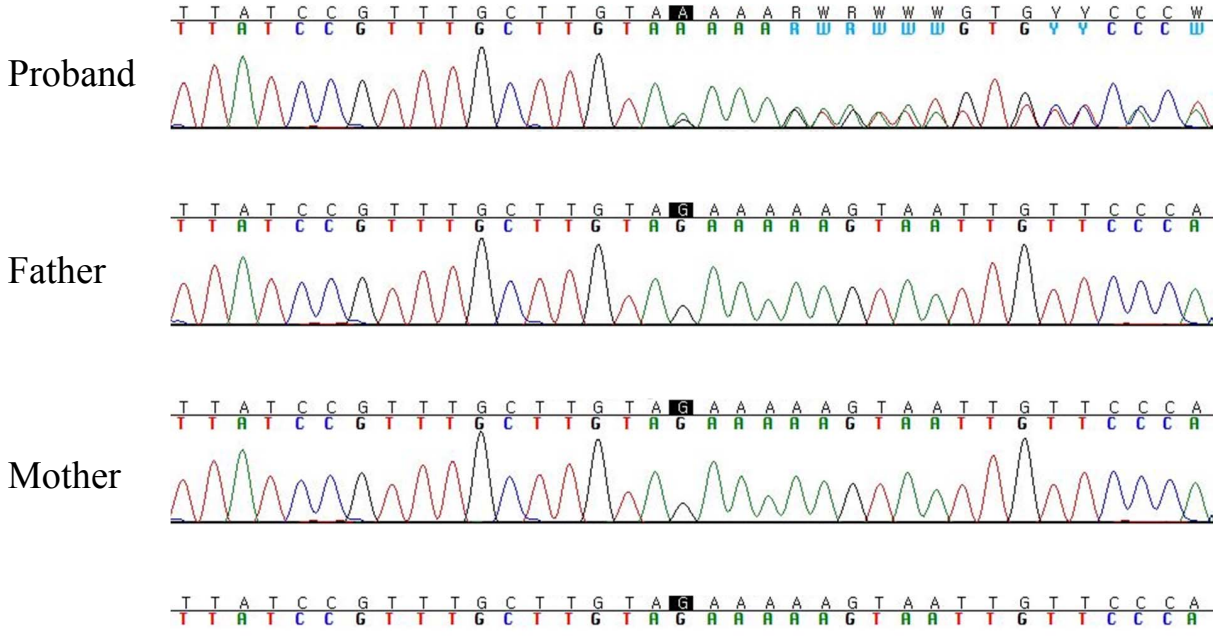

Brother

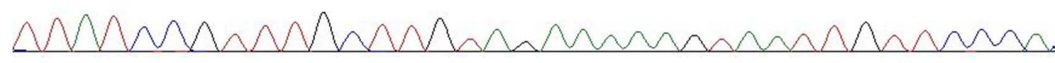

Fig. 3. Sanger sequencing of the TRPS1. DNA sequences of TRPS1 from the patient, parents and his younger brother are showing a de novo mutation in c.1801. 1802delGA (p.Arg601Lysfs*3) in exon 4 of the TRPS1 gene.

pain and increased fracture rate [5].

Although TRPS is reported in all ethnic groups, it is a rare disease [2]. TRPS is usually reported by pediatrics, dermatologist and orthopedics due to its characteristic clinical manifestations. In this case, patients showed only mild features of TRPS that included short stature and brachydactyly that did not affect 
the patient's social life. Moreover, his sparse hair was diagnosed as androgenetic alopecia by a dermatologist and he only complained of a non-specific fatigue and malaise. Several studies reported TRPS1 cases with endocrine disturbances, including diabetes mellitus, hypothyroidism, and growth hormone deficiency [6]. These endocrinal disorders may be the cause of fatigue or malaise, but endocrine functions were normal in our case to clearly explain his chronic fatigue and more studies are warranted to extrapolate it. Although the authors were suspicious of TRPS based on his characteristic features in his face (pearshaped nose) and extremities, we proceeded with the targeted exome sequencing as we were not confident of the diagnosis based on non-specific and mild symptoms of the patient.

The TRPS1 gene is highly expressed within the affected organs of the patients including cartilage, developing joints, hair follicles and the nasal regions [7]. In accordance to these clinical features, our patient also showed short stature, distinctive face and skeletal abnormalities. Interestingly our patient underwent temporomandibular joint surgery due to temporomandibular joint derangement and a recent study using Trps 1 knockout mice revealed that TRPS1 played an important role in development of temporomandibular joint that are in accordance to the clinical history of the patient [8]. Osteoporosis is rarely reported with low level of insulin growth factor and growth hormone which were unresponsive to the growth hormone substitutive therapy [9]. Unlike them, our patient showed osteopenia without hormonal abnormality, probably due to the mild phenotype and the efficacy of calcium and vitamin D needs to be validated.

TRPS1 gene constitutes of 7 exons and encodes polypeptide of 1,294 amino acids [10]. To date, more than 70 mutations in the TRPS1 gene have been reported [11]. TRPS1 is associated with mutation in one allele of TRPS1, which are believed to cause reduction of the concentration of TRPS1 protein in the nucleus (haploinsufficiency) [10]. There is no known hot spot in TRPS gene but according to a recent literature that reviewed 103 TRPS patients, most of the missense mutation was found in exon 6 or 7, and other mutations such as frameshift or nonsense mutation were usually found between exon 4 to 7 [5]. A recent study also showed that TRPS gene with nonsense mutations manifested a relatively mild phenotype [12] suggesting a hypothesis of reduced number of functional TRPS1 gene copies, consolidating the mild phenotype shown in our patient. On the other hand, missense mutation showed variable features ranging from mild phenotype of TRPS1 to severe phenotype of TRPS3, depending on the site of the mutation [12]. However, this molecular pathomechanism needs to be further elucidated.
Herein, a heterozygous frameshift mutation in the fourth exon of TRPS 1 was identified at the age of 20 due to his mild phenotype. Although the effect of the mutation on the expression of TRPS1 was unchecked, this frameshift mutation leads to premature stop codons downstream. In conclusion due to the rarity and variable phenotype of the disease, the diagnosis of TRPS can be challenging, but clinicians should be aware of this disease and its characteristic clinical features that will lead a higher rate of diagnosis and unravelling the patho-mechanism of TRPS.

\section{Acknowledgements}

This work was supported by the National Research Foundation of Korea (NRF) grant funded by the Korea government (Ministry of Science and ICT) (No. 2017R1C1B5076264).

\section{References}

1. Momeni P, Glöckner G, Schmidt O, von Holtum D, Albrecht B, Gillessen-Kaesbach $\mathrm{G}$, et al. Mutations in a new gene, encoding a zincfinger protein, cause tricho-rhino-phalangeal syndrome type I. Nat Genet 2000;24:71-4.

2. Maas S, Shaw A, Bikker H, Hennekam RC. Trichorhinophalangeal syndrome. In: Adam MP, Ardinger HH, Pagon RA, Wallace SE, eds. GeneReviews ${ }^{\circledR}$ [Internet]. Seattle (WA): University of Washington, 2017 Apr 20 [cited 2018 May 23]. [https://www.ncbi.nlm.nih.gov/books/ NBK425926/]

3. Napierala D, Sam K, Morello R, Zheng Q, Munivez E, Shivdasani RA, et al. Uncoupling of chondrocyte differentiation and perichondrial mineralization underlies the skeletal dysplasia in tricho-rhino-phalangeal syndrome. Hum Mol Genet 2008;17:2244-54.

4. Lüdecke HJ, Wagner MJ, Nardmann J, La Pillo B, Parrish JE, Willems PJ, et al. Molecular dissection of a contiguous gene syndrome: localization of the genes involved in the Langer-Giedion syndrome. Hum Mol Genet 1995;4:31-6.

5. Maas SM, Shaw AC, Bikker H, Lüdecke HJ, van der Tuin K, BaduraStronka $M$, et al. Phenotype and genotype in 103 patients with tricho-rhino-phalangeal syndrome. Eur J Med Genet 2015;58:27992.

6. Sohn YB, Ki CS, Park SW, Cho SY, Ko AR, Kwon MJ, et al. Clinical, biochemical, and genetic analysis of two Korean patients with trichorhinophalangeal syndrome type I and growth hormone deficiency. Ann Clin Lab Sci 2012;42:307-12.

7. Chen $L H$, Ning CC, Chao SC. Mutations in TRPS1 gene in trichorhinophalangeal syndrome type I in Asian patients. Br J Dermatol 2010; 
163:416-9.

8. Michikami I, Fukushi T, Honma S, Yoshioka S, Itoh S, Muragaki Y, et al. Trps1 is necessary for normal temporomandibular joint development. Cell Tissue Res 2012;348:131-40.

9. Stagi S, Bindi G, Galluzzi F, Lapi E, Salti R, Chiarelli F. Partial growth hormone deficiency and changed bone quality and mass in type I trichorhinophalangeal syndrome. Am J Med Genet A 2008;146A: 1598-604.

10. Giedion A, Burdea M, Fruchter Z, Meloni T, Trosc V. Autosomal-dom- inant transmission of the tricho-rhino-phalangeal syndrome. Report of 4 unrelated families, review of 60 cases. Helv Paediatr Acta 1973; 28:249-59.

11. Human gene mutation database $\left(\mathrm{HGMD}^{\circledR}\right)$. [http://www.hgmd.cf.ac. uk/ac/index.php]

12. Lüdecke HJ, Schaper J, Meinecke P, Momeni P, Gross S, von Holtum D, et al. Genotypic and phenotypic spectrum in tricho-rhino-phalageal syndrome types 1 and III. Am J Hum Genet 2001;68:81-91. 